Proceedings of the Edinburgh Mathematical Society (2003) 46, 747-766 (C)

DOI:10.1017/S0013091502000688 Printed in the United Kingdom

\title{
SYMPLECTIC CONNECTIONS WITH A PARALLEL RICCI CURVATURE
}

\author{
CHARLES BOUBEL \\ Université de Grenoble I, Institut Fourier (UMR 5582), BP 74, \\ 38402 Saint Martin d'Hères Cedex, France \\ (Charles.Boubel@ens-lyon.fr)
}

(Received 15 July 2002)

\begin{abstract}
A symplectic connection on a symplectic manifold, unlike the Levi-Civita connection on a Riemannian manifold, is not unique. However, some spaces admit a canonical connection (symmetric symplectic spaces, Kähler manifolds, etc.); besides, some 'preferred' symplectic connections can be defined in some situations. These facts motivate a study of the symplectic connections, inducing a parallel Ricci tensor. This paper gives the possible forms of the Ricci curvature on such manifolds and gives a decomposition theorem (linked with the holonomy decomposition) for them.
\end{abstract}

Keywords: affine connection; symplectic connection; Ricci curvature; Holonomy group; Einstein manifolds; pseudo-Riemannian manifolds

2000 Mathematics subject classification: Primary 53B05; 53B30; 53B35; 53C25; 53C55

\section{Introduction and motivation}

On a Riemannian or pseudo-Riemannian manifold, the Levi-Civita connection is defined. The symplectic analogue is the following. Let $(\mathcal{M}, \omega)$ be a symplectic manifold; a connection $D$ on $\mathcal{M}$ is said to be symplectic when

- $D$ is torsion free, i.e. for every vector field $x$ and $y, D_{x} y-D_{y} x-[x, y]=0$; and

- the symplectic form is parallel for $D$, i.e. $D \omega=0$.

With such a connection $D$ is associated its $(1,3)$-curvature tensor $R$ and its Ricci curvature tensor, here denoted by ric. Let us recall that ric is the bilinear symmetric form defined on each tangent space by $\operatorname{ric}(u, v)=\operatorname{tr} R(u, \cdot) v$. Unlike in the (pseudo-)Riemannian situation, the set of symplectic connections is an affine space of infinite dimension (see $\S 2$ (b) below). In some situations, however, there is a privileged one.

In the case in which $\mathcal{M}$ is a symmetric symplectic space, for instance (in the natural sense introduced in [10]; see also [2] for the symplectic case), it has a canonical connection, which is symplectic. This connection is symmetric, so in particular its Ricci curvature is parallel. 
In the case in which $\mathcal{M}$ is a (pseudo-)Riemannian manifold carrying a parallel symplectic form (e.g. a Kähler manifold), the Levi-Civita connection is also symplectic with respect to it.

On a general symplectic manifold, Bourgeois and Cahen introduced a variational principle distinguishing so-called 'preferred' symplectic connections in [6]. The corresponding field equations are

$$
D_{x} \operatorname{ric}(y, z)+D_{y} \operatorname{ric}(z, x)+D_{z} \operatorname{ric}(x, y)=0 .
$$

In particular, symplectic connections the Ricci curvature of which is parallel, i.e. such that $D$ ric $=0$, are therefore preferred. More generally, they have a specific interest in this theory and were recently studied by Cahen, Gutt and Rawnsley in [7]. Note also that the canonical connection of a symmetric symplectic manifold is thus preferred.

Riemannian manifolds the Ricci curvature of which is parallel, hereafter referred to as Ricci parallel, are, at least locally, products of Einstein manifolds. Pseudo-Riemannian Ricci-parallel manifolds admit an analogous, though slightly different, decomposition (see [5]). We show here a similar result for Ricci-parallel symplectic connections (see the Main Theorem in $\S 3$ ). It shall be noticed that the algebraic part of the result is the same as in the pseudo-Riemannian case, the geometrical consequence being weaker in general.

The structure of the article is as follows. After some lemmas and remarks are given in $\S 2$, the Main Theorem is stated and commented on in $\S 3$, and then proved in $\S 4$. Section 5 gives a refinement of the decomposition obtained in the Main Theorem and studies the subfactors. Finally, $\S 6$ provides some examples and final remarks.

Notation. On a symplectic manifold with a symplectic connection $(\mathcal{M}, \omega, D)$, we will denote by ric the Ricci tensor and by Ric the endomorphism induced by ric, i.e. the endomorphism such that $\operatorname{ric}(\cdot, \cdot)=\omega(\cdot, \operatorname{Ric} \cdot)$.

We denote by $H$ the holonomy group of $(\mathcal{M}, D)$, and classical Lie algebras are denoted using Gothic script. For example, if $p \in \mathcal{M}$, the symplectic Lie algebra $\mathfrak{s p}\left(\omega_{p}\right)$ is the algebra of the $\omega_{p}$-anti-self-adjoint endomorphisms of $T_{p} \mathcal{M}$; also $\mathfrak{h}$ denotes the Lie algebra of the holonomy group.

\section{Elementary facts about symplectic connections}

We need some basic facts in the following. Pointing them out here all in the same place will also make symplectic connections more familiar.

(a) As hinted at above, the properties satisfied by a symplectic connection $D$ are those that define the Levi-Civita connection of a Riemannian or pseudo-Riemannian metric $g$ if you replace $\omega$ by $g$. On any symplectic manifold, such a connection exists but it is not unique. The space of the symplectic connections associated with a given form $\omega$ is parametrized by $S^{3} T^{*} \mathcal{M}$; let us recall the following. 
Proposition 2.1. If $D$ is a symplectic connection on $(\mathcal{M}, \omega)$, then a connection $\Delta$ is symplectic if and only if

$$
\left[(x, y, z) \mapsto \omega\left(D_{x} y, z\right)-\omega\left(\Delta_{x} y, z\right)\right] \in S^{3} T^{*} \mathcal{M} .
$$

Proof. This is straightforward (see [9, p. 48]).

(b) The curvature tensor $R$ satisfies the usual algebraic properties:

- $R(x, y)=-R(y, x)$;

- $\omega(R(x, y) z, t)=\omega(R(x, y) t, z)$, i.e. all the $R(x, y)$ are $\omega$-anti-self-adjoint;

- $R(x, y) z+R(y, z) x+R(z, x) y=0$, which is the 'Bianchi identity'.

In the (pseudo-)Riemannian situation, an additional relation involving $R$ and the metric $g$ then follows:

$$
g(R(x, y) z, t)=g(R(z, t) x, y)
$$

It is not true with $R$ and $\omega, \omega$ being an alternate form. However, notice that, provided all the $R(x, y)$ for $x, y \in T_{p} \mathcal{M}$ are anti-self-adjoint with respect to a bilinear symmetric form $g$, we get (2.1) for $R$ and $g$, whether $g$ is degenerate or not. The proof does not need non-degeneracy (see, for example, $[\mathbf{1 1}$, p. 54]).

In particular, we get the following.

Lemma 2.2. If $g$ is a parallel symmetric bilinear form on $(\mathcal{M}, D), R$ and $g$ satisfy $(2.1)$.

Note. Relation (2.1) between $R$ and the metric $g$ is one of the essential tools giving the pseudo-Riemannian result [5]. So is it here: the Main Theorem is based on the fact that $R$ and ric satisfy (2.1), see the next remark.

(c) In the (pseudo-)Riemannian situation, ric is the only non-trivial invariant trace of $R$. In the symplectic case, there is a priori another one: $u, v \mapsto \operatorname{tr}_{\omega}[\omega(R(\cdot, \cdot) u, v)]$. However, it turns out that it is the same, up to a scalar; let us recall the (standard) result.

Lemma 2.3. If $(M, \omega, D)$ is a symplectic manifold with a symplectic connection,

$$
\operatorname{tr}_{\omega}[\omega(R(\cdot, \cdot) u, v)]=-2 \operatorname{ric}(u, v) .
$$

Proof. It follows from the Bianchi identity; as the result will be useful, let us recall its proof. 
Let $2 n$ be the dimension of $\mathcal{M}, p$ be a point in $\mathcal{M}$, and $\left(e_{i}\right)_{i=1}^{2 n}$ be a basis of $T_{p} \mathcal{M}$ such that $\omega=\sum_{i \leqslant n} e_{i}^{*} \wedge e_{n+i}^{*}$. For $a$ and $b$ in $T_{p} \mathcal{M}$,

$$
\begin{aligned}
\operatorname{tr}_{\omega}[\omega(R(\cdot, \cdot) a, b)] & =\sum_{i \leqslant n} \omega\left(R\left(e_{i}, e_{n+i}\right) a, b\right)-\omega\left(R\left(e_{n+i}, e_{i}\right) a, b\right) \\
& =2 \sum_{i \leqslant n} \omega\left(R\left(e_{i}, e_{n+i}\right) a, b\right) \\
& =2\left(\sum_{i \leqslant n} \omega\left(R\left(a, e_{n+i}\right) e_{i}, b\right)+\omega\left(R\left(e_{i}, a\right) e_{n+i}, b\right)\right) \\
& =2\left(\sum_{i \leqslant n} \omega\left(R\left(a, e_{n+i}\right) b, e_{i}\right)-\omega\left(R\left(a, e_{i}\right) b, e_{n+i}\right)\right) \\
& =-2 \operatorname{tr}[R(a, \cdot) b] \\
& =-2 \operatorname{ric}(a, b) .
\end{aligned}
$$

Important remark. The bilinear form ric is hence symmetric (this also holds for a Levi-Civita connection, but not in general for a torsion-free affine connection). In particular, Ric is $\omega$-anti-self-adjoint.

With Lemma 2.2, if ric is parallel, it implies also that (2.1) holds for ric and more generally for all the bilinear symmetric forms $\omega\left(\cdot, \operatorname{Ric} P\left(\operatorname{Ric}^{2}\right) \cdot\right)$, where $P$ is a polynomial.

(d) The endomorphism Ric in this framework. A final preliminary result is necessary before stating the theorem. It is some standard linear algebra but has to be precisely stated here.

Let $p$ be a point of $\mathcal{M}$; Ric being parallel, its minimal polynomial (i.e. the monic generator of the ideal of the polynomials $P$ of $\mathbb{R}[X]$ such that $P(\operatorname{Ric})=0$ ) is defined independently of the point. Now $\operatorname{Ric}_{\mid p} \in \mathfrak{s p}\left(\omega_{\mid p}\right)$, so we can apply the following well-known result to the complexified endomorphism $\operatorname{Ric}^{\mathbb{C}}$ of $T_{p} \mathcal{M} \otimes \mathbb{C}$.

Lemma 2.4. Let $(E, \omega)$ be a complex vector space endowed with a non-degenerate alternate form $\omega$ and $U$ in $\mathfrak{s p}(\omega)$. The minimal polynomial $\mu$ of $U$ then satisfies $\mu(X)=$ $\pm \mu(-X)$. There thus exists an $L \subset \mathbb{C}$ such that $L \cup(-L)=\{$ non-zero eigenvalues of $U\}$ and $L \cap(-L)=\emptyset$; with such an $L$,

$$
E=\operatorname{ker} U^{\alpha_{0}} \stackrel{\perp}{\oplus}\left(\underset{\lambda \in L}{\stackrel{\perp}{\oplus}}\left(\operatorname{ker}(U-\lambda \mathrm{Id})^{\alpha_{\lambda}} \oplus \operatorname{ker}(U+\lambda \mathrm{Id})^{\alpha_{\lambda}}\right)\right),
$$

where $\alpha_{\lambda}$ is the (common) power of $(X-\lambda)$ and $(X+\lambda)$ in $\mu$. The decomposition is orthogonal with respect to $\omega$ and each space $\operatorname{ker}(U \pm \lambda \mathrm{Id})$ is $\omega$-totally isotropic. Here $\alpha_{0}$ may be zero. 
Furthermore, Ric being real, its minimal polynomial $\mu$ is also invariant under complex conjugation; so taking, for example, $\Lambda=\{$ eigenvalues of Ric $\} \cap\left(\mathbb{R}^{+} \times i \mathbb{R}^{+}\right) \subset \mathbb{C}$, we get

$$
\mu=\prod_{\lambda \in \Lambda} P_{\lambda}^{\alpha_{\lambda}} \quad \text { with } \begin{cases}P_{0}=X & \text { appearing if } 0 \in \Lambda, \\ P_{\lambda}=(X-\lambda)(X+\lambda) & \text { if } \lambda \in \mathbb{R}^{*} \cup \mathrm{i} \mathbb{R}^{*}, \\ P_{\lambda}=(X-\lambda)(X+\lambda)(X-\bar{\lambda})(X+\bar{\lambda}) & \text { otherwise, }\end{cases}
$$

and the corresponding decomposition of $T_{p} \mathcal{M}$,

$$
T_{p} \mathcal{M}=\underset{\lambda \in \Lambda}{\oplus} \operatorname{ker}\left(P_{\lambda}^{\alpha_{\lambda}}(\mathrm{Ric})\right)
$$

Remark. This is the finest $\omega$-orthogonal decomposition of $T_{p} \mathcal{M}$ that is stable under the action of the centralizer of Ric. However, under this action and for example for $\lambda \in \mathbb{R}^{*}$,

- $\operatorname{ker}\left(P_{\lambda}^{\alpha_{\lambda}}(\operatorname{Ric})\right)=\operatorname{ker}(\operatorname{Ric}-\lambda \text { Id })^{\alpha_{\lambda}} \oplus \operatorname{ker}(\operatorname{Ric}+\lambda \operatorname{Id})^{\alpha_{\lambda}}$, each factor being stable but $\omega$-totally isotropic;

- $\operatorname{ker}(\operatorname{Ric}-\lambda \mathrm{Id})^{\alpha_{\lambda}}$ and $\operatorname{ker}(\operatorname{Ric}+\lambda \mathrm{Id})^{\alpha_{\lambda}}$ are irreducible if and only if $\alpha_{\lambda}=1$.

\section{The Main Theorem}

A Riemannian manifold with parallel Ricci curvature is, at least locally, a product of Einstein manifolds (this is only a remark (see [5, pp. 2, 3])). Let us recall that a manifold is Einstein if ric is proportional to the metric. In our situation, this notion makes no sense, since $\omega$ is alternate and ric symmetric.

Nevertheless, being a local product of Einstein Riemannian manifolds can be stated in other terms: ric is parallel and the minimal polynomial of Ric has simple roots in $\mathbb{C}$ (the roots are moreover real, $g$ being positive definite). That statement makes sense in our symplectic situation. Is it true? Yes, except possibly for the root zero. It is the same result as for a pseudo-Riemannian connection, the proof being quite different (see [5]).

Remark. Before stating the theorem, let us recall a straightforward fact linking holonomy-stable subspaces with some foliations. If $(\mathcal{M}, D)$ is a manifold endowed with a torsion-free connection $D$, if $p$ is a point of $\mathcal{M}$ and if the holonomy group stabilizes a subspace $A$ of $T_{p} \mathcal{M}$, then $A$ can be extended by parallel transport to a (parallel) distribution on $\mathcal{M}$. The connection being torsion free, this distribution is integrable; the leaves of the integral foliation are moreover totally geodesic.

The Main Theorem. Let $(M, \omega, D)$ be a symplectic manifold with a symplectic connection the Ricci curvature ric of which is parallel and $p \in \mathcal{M}$. Let $\mu$ be the minimal polynomial of Ric and $\mu=\prod_{\lambda \in \Lambda} P_{\lambda}^{\alpha_{\lambda}}$ the decomposition (2.2) of $\mu$ given in $\S 2$. For simplicity of the statement, we set $0 \in \Lambda$ and allow $\alpha_{0}$ to be null. Let us also denote by $M_{\lambda}$ the (parallel) distribution $\operatorname{ker}\left(P_{\lambda}^{\alpha_{\lambda}}(\mathrm{Ric})\right)$, by $\mathcal{M}_{\lambda}^{q}$ the integral leaf of $M_{\lambda}$ through a point $q$, and simply by $\mathcal{M}_{\lambda}$ the leaf $\mathcal{M}_{\lambda}^{p}$. Then we have the following. 
(i) For each $\lambda \neq 0, \alpha_{\lambda}$ is equal to 1 and $\alpha_{0} \leqslant 2$.

(ii) Setting, for each $\lambda, \omega_{\lambda}=\omega_{\mid T \mathcal{M}_{\lambda}}$ and $D_{\lambda}=D_{\mid T \mathcal{M}_{\lambda}},\left(\mathcal{M}_{\lambda}, \omega_{\lambda}, D_{\lambda}\right)$ is a symplectic manifold with a symplectic connection. Now, the unique local diffeomorphism $\mathcal{M} \rightarrow \prod_{\lambda} \mathcal{M}_{\lambda}$ preserving the integral foliations of the $M_{\lambda}$ and equal to identity on the $\mathcal{M}_{\lambda}$ identifies, in a suitable neighbourhood of $p, \mathcal{M}$ with $\prod_{\lambda} \mathcal{M}_{\lambda}$. With this identification,

$$
(\mathcal{M}, \omega, D) \simeq\left(\prod_{\lambda} \mathcal{M}_{\lambda}, \prod_{\lambda} \omega_{\lambda},\left(\prod_{\lambda} D_{\lambda}\right)+S\right)
$$

with $S$ a (1,2)-tensor on $\mathcal{M}$. Moreover, $D$ and $\prod_{\lambda} D_{\lambda}$ have the same Ricci curvature and $S$ satisfies the following properties.

- $\omega(S(\cdot, \cdot), \cdot)$ is completely symmetric.

- $S$ is a section of $\pi_{0}^{*}\left(T_{2}^{1} \mathcal{M}_{0}\right)$, where $\pi_{0}$ is defined, at each point $q$, as the canonical projection $T_{q} \mathcal{M}=\oplus_{\lambda} T_{q} \mathcal{M}_{\lambda}^{q} \rightarrow T_{q} \mathcal{M}_{0}^{q}$.

- $\forall(x, y) \in T_{q} \mathcal{M}, \operatorname{tr}\left[z \mapsto D_{z} S(x, y)\right]-\operatorname{tr}[z \mapsto S(x, S(y, z))]=0$.

- $\operatorname{Im} \operatorname{Ric} \subset \operatorname{ker} S$,

the last property being a consequence of the third one.

We prove the Main Theorem in $\S 4$. We make some remarks here.

(a) As the decomposition

$$
T_{p} \mathcal{M}=\oplus_{\lambda} M_{\lambda}
$$

is unique, so is the collection $\left(\left(\mathcal{M}_{\lambda}, \omega_{\lambda}, D_{\lambda}\right)_{\lambda \in \Lambda}, S\right)$.

(b) Once it is supposed that ric is parallel, the first point of the theorem is a purely 'pointwise' consequence of the algebraic properties of the curvature tensor $R$. The second one is a consequence of an adaptation of de Rham's decomposition theorem of Riemannian manifolds (see Proposition 3.1 below).

Point (i) will then give information on the factors $\mathcal{M}_{\lambda}$ given by point (ii); see $\S 5$, in particular Proposition 5.6 in $\S 5.2$.

Proposition 3.1. Let $(\mathcal{M}, \omega, D)$ be a symplectic manifold with a symplectic connection and $p \in \mathcal{M}$. Suppose that the restricted holonomy group $H^{0}$ preserves an $\omega$-orthogonal decomposition

$$
T_{p} \mathcal{M}=\underset{0 \leqslant i \leqslant k}{\oplus} M_{i}
$$

of $T_{p} \mathcal{M}$. Then for each $i, M_{i}$ induces by parallel transport a parallel, thus integrable, distribution on $\mathcal{M}$, also denoted by $M_{i}$.

Let $\left(\mathcal{M}_{i}\right)$ be the integral manifold through $p$ of the distribution $M_{i}$. Then

(i) the $\left(\mathcal{M}_{i}, \omega_{i}, D_{i}\right)=\left(\mathcal{M}_{i}, \omega_{\mid T \mathcal{M}_{i}}, D_{\mid T \mathcal{M}_{i}}\right)$ are symplectic manifolds with a symplectic connection; 
(ii) the unique local diffeomorphism preserving the foliations induced by the $M_{i}$ and equal to identity on the $\mathcal{M}_{i}$ identifies, in a suitable neighbourhood of $p, \mathcal{M}$ to $\prod_{i} \mathcal{M}_{i}$; on this neighbourhood, $\omega=\prod_{i} \omega_{i}$;

(iii) with this local identification $\mathcal{M} \simeq \prod_{i} \mathcal{M}_{i}$, there is $S$ a (unique) (1,2)-tensor on $\mathcal{M}$ such that $D=\left(\prod_{i} D_{i}\right)+S$.

Moreover, $S$ satisfies the following conditions:

- $\omega(S(\cdot, \cdot), \cdot)$ is symmetric;

- $S=\sum_{i} S^{i}$ where each $S^{i}$ is a section of $\pi_{i}^{*}\left(T_{2}^{1} \mathcal{M}_{i}\right)$, where $\pi_{i}$ is defined, at each point $q$, as the canonical projection $T_{q} \mathcal{M}=\oplus_{j} T_{q} \mathcal{M}_{j}^{q} \rightarrow T_{q} \mathcal{M}_{i}^{q}$;

- for each $i$ and each $q \in \mathcal{M}_{i}, S_{q}^{i}=0$, i.e. $S_{\mid\left(T \mathcal{M}_{i}\right)^{2}}$ is null on $\mathcal{M}_{i}$.

This proposition is, adapted to a symplectic connection, the local (and easy) part of de Rham's Theorem. Its proof will also be given in $\S 4$. Two points of the Riemannian theorem fail to be true here.

- The result is weaker - and a little deceptive-because $(\mathcal{M}, D)$ is not a product for the affine structure: $\mathcal{M} \simeq \prod_{i}\left(\mathcal{M}_{i}, \omega_{i}\right)$ but $D \neq \prod_{i} D_{i}$. This is due to the nonuniqueness of a symplectic connection on a symplectic manifold.

- For a Riemannian manifold $\mathcal{M}, T_{p} \mathcal{M}$ is the sum of a trivial subrepresentation of $H$ and of a sum of irreducible subrepresentations; a consequence is the uniqueness of this decomposition. It is not the case here, since $T_{p} \mathcal{M}$ may admit reducible indecomposable factors. So there does not generally exist any canonical decomposition of $T_{p} \mathcal{M}$ under the action of $H$ (or of $H^{0}$ ).

Nevertheless, when $(\mathcal{M}, \omega, D)$ is a symmetric symplectic space, a quite unexpected decomposition result holds (see [4, Theorems 2.3 and 2.12]).

(c) In general, the local symplectomorphism $(\mathcal{M}, \omega) \rightarrow \prod_{\lambda}\left(\mathcal{M}_{\lambda}, \omega_{\lambda}\right)$ of the Main Theorem is not an isomorphism of affine structure from $(\mathcal{M}, D)$ on $\prod_{\lambda}\left(\mathcal{M}_{\lambda}, D_{\lambda}\right)$. However, it $i s$ one when Ric is non-degenerate; the following decomposition then holds.

Corollary 3.2. Let $(M, \omega, D)$ be a symplectic manifold with a symplectic connection, the Ricci curvature ric of which is parallel and non-degenerate. Let $\mu$ be the minimal polynomial of Ric and let $\mu=\prod_{\lambda \in \Lambda} P_{\lambda}^{\alpha_{\lambda}}$ be the decomposition (2.2) of $\mu$ given in $\S 2$. Then

(i) for each $\lambda, \alpha_{\lambda}$ is equal to 1 (and $0 \notin \Lambda$ since ric is non-degenerate);

(ii) there exists a unique family $\left(\left(\mathcal{M}_{\lambda}, \omega_{\lambda}, D_{\lambda}\right)\right)_{\lambda \in \Lambda}$ of symplectic manifolds with a symplectic connection such that

- for each $\lambda$, the minimal polynomial of $\operatorname{Ric}_{\mathcal{M}_{\lambda}}$ is $P_{\lambda}$,

$-(\mathcal{M}, \omega, D)$ is locally affinely symplectomorphic to $\prod_{\lambda}\left(\mathcal{M}_{\lambda}, \omega_{\lambda}, D_{\lambda}\right)$; 
(iii) if $(\mathcal{M}, \omega, D)$ is moreover geodesically complete and simply connected, the isomorphism of point (ii) is global.

Proof. Points (i) and (ii) are simply the case ' $\alpha_{0}=0, \mathcal{M}_{0}$ reduced to a point' of the Main Theorem: then $S=0$, which gives points (i) and (ii). Point (iii) is an immediate consequence of the global part of Wu's Theorem (see [12] and $\S 5.1$ in this paper) applied to the pseudo-Riemannian manifold $(\mathcal{M}$, ric $)$.

Alternative, self-contained proof. We can also easily understand autonomously the reason it works. Indeed in that case, $(\mathcal{M}$, ric) turns out to be a pseudo-Riemannian manifold (which is moreover Einstein with constant 1 by definition). Ric being parallel, the decomposition

$$
T_{p} \mathcal{M}=\underset{\lambda \in \Lambda}{\stackrel{\perp}{\oplus}} \operatorname{ker}\left(P_{\lambda}^{\alpha_{\lambda}}(\mathrm{Ric})\right)
$$

is preserved by the action of the holonomy group. Applying Wu's Theorem, the pseudoRiemannian generalization of de Rham's Theorem (see [12]), we get that $\mathcal{M}$ is isomorphic to the Riemannian product of the factors $\mathcal{M}_{\lambda}$. Besides, the symplectic connection $D$ is torsion free and satisfies $D$ ric $=0$, so it is the Levi-Civita connection of the metric ric. Consequently, the Riemannian product is also an affine morphism $(\mathcal{M}, D) \simeq \prod_{\lambda}\left(\mathcal{M}_{\lambda}, D_{\lambda}\right)$.

(d) Conversely, if $\left(\mathcal{M}_{i}, \omega_{i}, D_{i}\right)_{i=0}^{k}$ are symplectic manifolds with Ricci-parallel symplectic connections, with $\operatorname{Ric}_{i}$ non-degenerate except for $i=0$, then a manifold of the type

$$
\left(\prod_{i}\left(\mathcal{M}_{i}, \omega_{i}\right), \prod_{i} D_{i}+S\right)
$$

with $S$ satisfying the properties listed in the Main Theorem, is Ricci parallel. This is an immediate consequence of Proposition 2.1 combined with Lemma 4.3 below.

\section{Proof of the Main Theorem}

Proof of Proposition 3.1 of $\S 3$. We have to check that the Riemannian proof (see [8, pp. 179 onwards]) remains valid or can be adapted at each step. Let us do it for $k=2$, the general case then comes by induction. We denote $M_{1}, \mathcal{M}_{1}, M_{2}$ and $\mathcal{M}_{2}$ by $A, \mathcal{A}, B$ and $\mathcal{B}$, respectively. For another point $q$ of $\mathcal{M}, \mathcal{A}_{q}$ (respectively, $\mathcal{B}_{q}$ ) will stand for the integral leaf of $A$ (respectively, $B$ ) through $q$.

(i) At $p, \omega_{\mid A}$ and $\omega_{\mid B}$ are non-degenerate. Now $\mathcal{A}$ and $\mathcal{B}$ being integral leaves of parallel distributions and $\omega$ being parallel, $\omega_{\mid T \mathcal{A}}$ and $\omega_{\mid T \mathcal{B}}$ are non-degenerate; let us denote them by $\omega^{\mathcal{A}}$ and $\omega^{\mathcal{B}}$. $\mathcal{A}$ is totally geodesic, so the restriction $D^{\mathcal{A}}$ to $T \mathcal{A}$ of the connection $D$ is the connection induced by $D$ on the submanifold $\mathcal{A}$. Hence similarly for $\mathcal{B}$. Eventually, as $D \omega=0, D^{\mathcal{A}} \omega^{\mathcal{A}}=0$ and $\left(\mathcal{A}, \omega^{\mathcal{A}}, D^{\mathcal{A}}\right)$ is (locally) a symplectic submanifold of $\mathcal{M}$, with a symplectic connection (hence also for $\left(\mathcal{B}, \omega^{\mathcal{B}}, D^{\mathcal{B}}\right)$ ). 
(ii) The fact that $\mathcal{M}$ is locally canonically diffeomorphic to $\mathcal{A} \times \mathcal{B}$ is obvious and purely differential (see the lemma on p. 182 of [8] for a formal proof). We can then take local coordinates of $\mathcal{M}$ of the form $\left(\left(a_{i}\right)_{i=1}^{d_{\mathcal{A}}},\left(b_{i}\right)_{i=1}^{d_{\mathcal{B}}}\right)$ such that, at every point $q$,

$$
A_{q}=\operatorname{span}\left(\frac{\partial}{\partial a_{i}}\right)_{i=1}^{d_{\mathcal{A}}} \text { and } B_{q}=\operatorname{span}\left(\frac{\partial}{\partial b_{i}}\right)_{i=1}^{d_{\mathcal{B}}}
$$

To prove that $\omega$ is equal to the product form $\omega^{\mathcal{A}} \times \omega^{\mathcal{B}}$, we have to show: for each $(i, j, k)$,

$$
L_{\partial / \partial b_{i}}\left[\omega\left(\frac{\partial}{\partial a_{j}}, \frac{\partial}{\partial a_{k}}\right)\right]=0 .
$$

It follows [8, Proposition 5.2, p. 182] from the fact that $D$ is torsion free. Indeed for each $(i, j), D_{\partial / \partial b_{i}}\left(\partial / \partial a_{j}\right)=D_{\partial / \partial a_{j}}\left(\partial / \partial b_{i}\right)$. Now, as the distributions $A$ and $B$ are parallel, $D_{\partial / \partial b_{i}}\left(\partial / \partial a_{j}\right) \in A$ and $D_{\partial / \partial a_{j}}\left(\partial / \partial b_{i}\right) \in B$. Thus

$$
D_{\partial / \partial b_{i}}\left(\frac{\partial}{\partial a_{j}}\right)=D_{\partial / \partial a_{j}}\left(\frac{\partial}{\partial b_{i}}\right)=0 .
$$

Then

$$
\begin{aligned}
& L_{\partial / \partial b_{i}}\left[\omega\left(\frac{\partial}{\partial a_{j}}, \frac{\partial}{\partial a_{k}}\right)\right] \\
& =\underbrace{\left(D_{\partial / \partial b_{i}} \omega\right)}_{=0}\left(\frac{\partial}{\partial a_{j}}, \frac{\partial}{\partial a_{k}}\right)+\omega(\underbrace{\left.D_{\partial / \partial b_{i}\left(\frac{\partial}{\partial a_{j}}\right)}\right)}_{=0}, \frac{\partial}{\partial a_{k}})+\omega(\frac{\partial}{\partial a_{j}}, \underbrace{D_{\partial / \partial b_{i}}\left(\frac{\partial}{\partial a_{j}}\right)}_{=0})=0 .
\end{aligned}
$$

(iii) The properties of $\boldsymbol{S}$. The product connection $D^{\mathcal{A}} \times D^{\mathcal{B}}$ on $\mathcal{A} \times \mathcal{B}$ is a symplectic connection. Indeed, the local product structure of $(\mathcal{M}, \omega)$ induces a local diffeomorphism between $\mathcal{A}_{p}$ and $\mathcal{A}_{q}$ for each point $q$, preserving moreover $\omega$ and mapping $D^{\mathcal{A}}$ on $\left(D^{\mathcal{A}} \times\right.$ $\left.D^{\mathcal{B}}\right)_{\mid T \mathcal{A}_{q}}$ by definition of $D^{\mathcal{A}} \times D^{\mathcal{B}}$. As $D^{\mathcal{A}} \omega=0$ on $\mathcal{A}_{p},\left(D^{\mathcal{A}} \times D^{\mathcal{B}}\right)_{\mid T \mathcal{A}_{q}} \omega=0$. So by Proposition 2.1 , there exists a $(1,2)$-tensor $S$ on $\mathcal{A}_{q}$ such that

- $D_{\mid T \mathcal{A}_{q}}=\left(D^{\mathcal{A}} \times D^{\mathcal{B}}\right)_{\mid T \mathcal{A}_{q}}+S^{\mathcal{A}_{q}}$,

- $\omega\left(S^{\mathcal{A}_{q}}(\cdot, \cdot), \cdot\right)$ is symmetric.

Now, as shown above, at any point of $\mathcal{M}$ and for any indexes $(i, j), D_{\partial / \partial b_{i}}\left(\partial / \partial a_{j}\right)=$ $D_{\partial / \partial a_{j}}\left(\partial / \partial b_{i}\right)=0$. The same equality is true for the product connection $D^{\mathcal{A}} \times D^{\mathcal{B}}$, by its definition. So, for all $(i, j), S\left(\partial / \partial a_{j}, \partial / \partial b_{i}\right)=0$. Therefore, as $S$ is a tensor, $S_{\mid q}$ can be written, pointwise at each point $q$, as

$$
S_{\mid q}=\left(\pi_{A}\right)^{*}\left(S_{\mid q}^{\mathcal{A}_{q}}\right)+\left(\pi_{B}\right)^{*}\left(S_{\mid q}^{\mathcal{B}_{q}}\right)
$$

where $\pi_{A}$ is the projection $T_{q} \mathcal{M}=A_{q} \oplus B_{q} \rightarrow A_{q}$ and similarly for $\pi_{B}$. The result follows. 
In addition to Proposition 3.1, we will also use three more lemmas. Point (i) of the Main Theorem is a consequence of an essential technical lemma we state here.

Lemma 4.1. Let $p$ be a point of $\mathcal{M}, U \in \mathfrak{s p}\left(\omega_{\mid p}\right)$ (i.e. $U$ is an $\omega$-anti-self-adjoint endomorphism of $\left.T_{p} \mathcal{M}\right)$, commuting with all the $R(x, y)$ for $x, y \in T_{p} \mathcal{M}$. Let us take $a, b \in T_{p} \mathcal{M}$ with $b \in \operatorname{Im} U$. The bilinear form $\omega(R(\cdot, \cdot) a, b)$ is skew-symmetric; let us denote by $A_{a, b}$ the $\omega$-self-adjoint endomorphism such that $\omega(R(\cdot, \cdot) a, b)=\omega\left(\cdot, A_{a, b} \cdot\right)$. Then

$$
A_{a, b}=-U \circ R(a, c)=-R(a, c) \circ U, \quad \text { where } c \text { is any antecedent of } b \text { by } U .
$$

Proof of Lemma 4.1. Let us simply write here $A=A_{a, b}$ and let us take $c$ such that $U c=b$. As $U \in \mathfrak{s p}\left(\omega_{\mid p}\right)$, the bilinear form $u:(x, y) \mapsto \omega(x, U y)$ is symmetric; as all the $R(x, y)$ are supposed to commute with $U$, notice they are all $u$-anti-self-adjoint:

$$
\begin{aligned}
u(R(x, y) z, t) & =\omega(R(x, y) z, U t) \\
& =\omega(R(x, y) U t, z) \\
& =\omega(U R(x, y) t, z) \\
& =-\omega(z, U R(x, y) t) \\
& =-u(z, R(x, y) t) .
\end{aligned}
$$

Consequently, by Lemma 2.2 of $\S 2,(2.1)$ holds for $u$ :

$$
\forall x, y, z, t, \quad u(R(x, y) z, t)=u(R(z, t) x, y) .
$$

To prove the lemma it is sufficient to check that

$$
\forall x, y \in T_{p} \mathcal{M}, \quad \omega(x, A y)=\omega(x, U(R(a, c) y)) .
$$

Let $x, y$ be any two vectors in $T_{p} \mathcal{M}$. Then

$$
\begin{aligned}
\omega(x, A y) & =\omega(R(x, y) a, b) & & \\
& =\omega(R(x, y) a, U c) & & \\
& =u(R(x, y) a, c) & & (\text { by definition of } u) \\
& =u(R(a, c) x, y) & & (\text { by }(2.1)) \\
& =-u(x, R(a, c) y) & & (R(a, c) \text { being } u \text {-anti-self-adjoint) } \\
& =-\omega(x, U R(a, c) y) & & \text { (by definition of } u) .
\end{aligned}
$$

Let us also recall the following standard remark.

Lemma 4.2. Let $E$ be a real or complex vector space, $\langle\cdot, \cdot\rangle$ a reflexive, i.e. symmetric or skew-symmetric form on $E$ and $U$ a $\langle\cdot, \cdot\rangle$-anti-self-adjoint endomorphism of $E$. Let $U=S+T$ be the decomposition of $U$ into its semi-simple and nilpotent parts the unique such decomposition with $S T=T S)$. Then $S$ and $T$ are $\langle\cdot, \cdot\rangle$-anti-self-adjoint. 
For point (ii) we will also need the following (standard) result.

Lemma 4.3. Let $D$ and $D^{\prime}$ be two symplectic connections on a symplectic manifold $(\mathcal{M}, \omega)$ and let $S$ be the tensor such that $D^{\prime}=D+S$. Let us denote by ric and ric' the Ricci curvatures induced by $D$ and $D^{\prime}$, respectively, and by $S_{x}$ the endomorphism $S(x, \cdot)$. Then

$$
\operatorname{ric}^{\prime}(x, y)=\operatorname{ric}(x, y)-\operatorname{tr}\left[z \mapsto\left(D_{z} S\right)(x, y)\right]+\operatorname{tr} S_{x} S_{y} .
$$

Proof. It is sufficient to give the proof with vector fields which are coordinate vector fields for some normal coordinate system at some point $p$ in $\mathcal{M}$. For two distinct such vectors $u$ and $v, D_{u} v=D_{v} u$, and, at $p, D_{u} v=0$. With such vectors, a straightforward computation gives

$$
\begin{aligned}
R^{\prime}(x, z) y & =(D+S)_{z}(D+S)_{x} y-(D+S)_{x}(D+S)_{z} y \\
& =R(x, z) y+\left(D_{x} S\right)_{z} y-\left(D_{z} S\right)_{x} y+S_{x} S_{z} y-S_{z} S_{x} y
\end{aligned}
$$

So

$$
\operatorname{ric}^{\prime}(x, y)=\operatorname{ric}(x, y)+\operatorname{tr}\left[z \mapsto\left(D_{x} S\right)_{z} y-\left(D_{z} S\right)_{x} y-S_{x} S_{z} y+S_{z} S_{x} y\right] .
$$

Now we have the following.

- $\left[z \mapsto S_{z} S_{x} y\right]=[z \mapsto S(z, S(x, y))]=S_{S(x, y)}$. But $\omega(S(\cdot, \cdot), \cdot)$ is symmetric, so in particular,

$$
\omega\left(S_{u} v, w\right)=\omega(S(u, v), w)=\omega(S(u, w), v)=-\omega(v, S(u, w))=-\omega\left(v, S_{u} w\right)
$$

so the $S_{u}$ are in $\mathfrak{s p}(\omega)$, and are thus trace free. So $\operatorname{tr}\left[z \mapsto S_{z} S_{x} y\right]=0$.

- For the same reason, $\operatorname{tr}\left(D_{x} S\right)_{y}=0$. So by symmetry of $S, \operatorname{tr}\left[z \mapsto\left(D_{x} S\right)_{z} y\right]=$ $\operatorname{tr}\left[\left(D_{x} S\right)_{y}\right]=0$.

- By symmetry of $S, S_{x} S_{z} y=S_{x} S_{y} z$.

The result follows.

Proof of the theorem. (i) Let Ric $=S+T$ be the decomposition of Ric into its semi-simple and nilpotent parts. As $S$ and $T$ are polynomials in Ric, they are themselves parallel. Let $p$ be a point in $\mathcal{M}$, and let us consider the endomorphism $T$ acting on $T_{p} \mathcal{M}$. Let us take $b \in \operatorname{Im} T$, say $b=T(c)$. By Lemma $4.2, T \in \mathfrak{s p}\left(\omega_{p}\right) ; T$ being parallel, it commutes with all the $R(x, y)$ for $x, y \in T_{p} \mathcal{M}$, we can therefore apply Lemma 4.1. Combined with Lemma 2.3 this gives

$$
\begin{aligned}
& \forall a \in T_{p} \mathcal{M}, \operatorname{ric}(a, b)=-\frac{1}{2} \operatorname{tr}_{\omega}[R(\cdot, \cdot) a, b] \quad \text { (Lemma 2.3) } \\
& =-\frac{1}{2} \operatorname{tr}[R(a, c) \circ T] \quad(\text { Lemma 4.1). }
\end{aligned}
$$

But $T$ is parallel so it commutes with $R(a, c)$; thus, $T$ being nilpotent, so is $R(a, c) \circ T$. So $R(a, c) \circ T$ is trace free, which means that $\forall a \in T_{p} \mathcal{M}, \operatorname{ric}(a, b)=0$, that is to say $b \in$ ker Ric. So we get (at any point)

$\operatorname{Im} T \subset$ ker Ric . 
That is the desired result. Indeed if $\mu$ is the minimal polynomial of Ric, you can write

$$
\mu=X^{\alpha_{0}} \prod_{\lambda}(X-\lambda)^{\alpha_{\lambda}},
$$

where $\lambda$ runs over the set of the non-zero eigenvalues of Ric and where $\alpha_{0}$ is the - possibly null-power of $X$ in $\mu$. Then Ric is non-degenerate on $\operatorname{ker}\left[\prod_{\lambda}(\operatorname{Ric}-\lambda \mathrm{Id})^{\alpha_{\lambda}}\right]$ so on this space $\operatorname{Im} T=\{0\}$, i.e. $T=0$, i.e. all the $\alpha_{\lambda}$ for $\lambda$ a non-zero eigenvalue of Ric are 1. On $\operatorname{ker} S=\operatorname{ker} \operatorname{Ric}^{\alpha_{0}}, T$ is equal to $\operatorname{Ric}$ so $\operatorname{Im} T \subset \operatorname{ker} T$, i.e. $\alpha_{0} \leqslant 2$.

(ii) The decomposition and the tensor $S$ are given by Proposition 3.1. Let us denote by ric $^{\prime}$ the Ricci curvature of the product connection $D-S$ and let us prove that ric ${ }^{\prime}=$ ric. Let us take $\left(x_{i}^{\lambda}\right)_{i=1}^{n_{\lambda}}$, where $n_{\lambda}=\operatorname{dim} \mathcal{M}_{\lambda}$, local coordinates on each $\mathcal{M}_{\lambda}$, in a neighbourhood of $p ;\left(\left(x_{i}^{\lambda}\right)_{i=1}^{n_{\lambda}}\right)_{\lambda \in \Lambda}$ are coordinates of $\mathcal{M}$ in a neighbourhood of $p$. Let $q$ be a point of $\mathcal{M}$ in such a neighbourhood and $q_{\lambda}$ its projection on $\mathcal{M}_{\lambda}$, for any $\lambda \in \Lambda$.

- From the definition of the product connection, it follows that

$$
\operatorname{ric}_{\mid q}^{\prime}\left(\frac{\partial}{\partial x_{i}^{\lambda}}, \frac{\partial}{\partial x_{j}^{\lambda}}\right)=\operatorname{ric}_{q_{\lambda}}^{\prime}\left(\frac{\partial}{\partial x_{i}^{\lambda}}, \frac{\partial}{\partial x_{j}^{\lambda}}\right)
$$

for all $i, j$.

- By (4.1) applied to the distributions $M_{\lambda}$ and the coordinate vectors $\partial / \partial x_{i}^{\lambda}$,

$$
\lambda \neq \lambda^{\prime} \Rightarrow \forall i, j, D_{\partial / \partial x_{i}^{\lambda^{\prime}}} \partial / \partial x_{j}^{\lambda}=0 .
$$

In particular, the vector fields $\partial / \partial x_{i}^{\lambda}$ are $D$-parallel along any path tangent to $\oplus_{\lambda^{\prime} \neq \lambda} M_{\lambda^{\prime}}$. Now $q$ and $q_{\lambda}$ are joined by such a path so, as ric is $D$-parallel by assumption, the parallel transport along this path gives

$$
\operatorname{ric}_{\mid q}\left(\frac{\partial}{\partial x_{i}^{\lambda}}, \frac{\partial}{\partial x_{j}^{\lambda}}\right)=\operatorname{ric}_{\mid q_{\lambda}}\left(\frac{\partial}{\partial x_{i}^{\lambda}}, \frac{\partial}{\partial x_{j}^{\lambda}}\right)
$$

for all $i, j$.

Besides, by construction, $D$ and $D-S$ coincide on each $T \mathcal{M}_{\lambda}$, so also do their Ricci curvatures ric and ric $^{\prime}$ and therefore, for any $\lambda$ and any $(i, j)$,

$$
\operatorname{ric}_{\mid q}^{\prime}\left(\frac{\partial}{\partial x_{i}^{\lambda}}, \frac{\partial}{\partial x_{j}^{\lambda}}\right)=\operatorname{ric}_{\mid q}\left(\frac{\partial}{\partial x_{i}^{\lambda}}, \frac{\partial}{\partial x_{j}^{\lambda}}\right) .
$$

Finally, as the $M_{\lambda}$ are mutually ric-orthogonal (by definition of the $M_{\lambda}$ ) and ric'orthogonal (by definition of the product connection), ric $=$ ric $^{\prime}$.

The first property of $S$ comes from Proposition 2.1. Let us prove the factorization of $S$. On the similar integral manifold $\mathcal{M}_{\lambda}^{q}$ through any $q$, for $\lambda \neq 0$, the non-degenerate bilinear form $\operatorname{ric}_{\mathcal{M}_{\lambda}^{q}}$ is parallel for the product connection $\left(\prod_{\lambda} D_{\lambda}\right)_{\mid T \mathcal{M}_{\lambda}^{q}}$ and for the original connection $D$ of $\mathcal{M}$. So these connections are both equal to the Levi-Civita connection of $\operatorname{ric}_{\mathcal{M}_{\lambda}^{q}}$. So $S_{\mid T \mathcal{M}_{\lambda}^{q}}=0$. This gives, together with Proposition 3.1, the factorization of $S$. 
After Lemma 4.3 above in this section, $S$ satisfies the third property if and only if $D$ and $D-S$ have the same Ricci curvature, which has been shown.

This implies finally that $\operatorname{Im} \operatorname{Ric} \subset \operatorname{ker} S$. To see this, we provide the following claim.

Claim 4.4. Let $D^{\prime}$ be a symplectic connection on some integral manifold $\mathcal{M}_{0}^{q}$ of $M_{0}=$ ker Ric ${ }^{2}$ through some point $q$, inducing the same Ricci curvature as $D$, and let $S^{\prime}$ be the tensor such that $D^{\prime}=D+S^{\prime}$. Then $\operatorname{Im} \operatorname{Ric} \subset \operatorname{ker} S^{\prime}$.

Let us indeed choose normal coordinates based at $q$. Then, for $(x, y, z)$ any triple of coordinate vectors and ric being parallel,

$$
2 \operatorname{ric}\left(D_{x}^{\prime} y, z\right)=L_{x} \operatorname{ric}(y, z)+L_{y} \operatorname{ric}(x, z)+L_{z} \operatorname{ric}(x, y),
$$

by the same computations as those that give the expression of the Levi-Civita connection of a metric $g$. So $\operatorname{ric}\left(D_{x}^{\prime} y, z\right)$ is fixed, i.e. is equal to $\operatorname{ric}\left(D_{x} y, z\right)$. Therefore, $\operatorname{ric}\left(S^{\prime}(\cdot, \cdot), \cdot\right)=$ 0 or, equivalently, $\omega\left(S^{\prime}(\cdot, \cdot)\right.$, Im Ric $)=0$ by definition of Ric. By symmetry of $S^{\prime}$, this is again equivalent to $S^{\prime}(\operatorname{Im} \operatorname{Ric}, \cdot)=0$. So the claim holds, which completes the proof.

\section{Ricci decomposition and holonomy decomposition}

\subsection{A refinement of the decomposition given by the Main Theorem}

The decomposition of $(\mathcal{M}, \omega, D)$ appearing in the Main Theorem may be refined. Let us introduce a definition.

Definition 5.1. A pseudo-Riemannian manifold is said to be weakly irreducible if the holonomy group does not stabilize any non-degenerate proper subspace.

Remark. Obviously, the holonomy representation is weakly irreducible if and only if it does not admit any decomposition into a direct orthogonal sum of stable subspaces.

De Rham's Theorem on the decomposition of the Riemannian manifolds into a product of irreducible ones admits a pseudo-Riemannian generalization, in fact nearly the best that could be expected, i.e. the elementary factors are weakly irreducible. We recall the result of [12, Appendix 1, p. 389].

Theorem 5.2 (de Rham, Wu). Let $(\mathcal{M}, g)$ be a geodesically complete, simply connected Riemannian or pseudo-Riemannian manifold and $p \in \mathcal{M}$. We suppose that the maximal trivial subspace $M_{p}^{0}$ of $H$ in $T_{p} \mathcal{M}$ is non-degenerate. Then

(i) $T_{p} \mathcal{M}$ admits a decomposition, unique up to order,

$$
T_{p} \mathcal{M}=\underset{0 \leqslant i \leqslant k}{\stackrel{\perp}{\oplus}} M_{p}^{i}
$$

and $H$ the decomposition, $H \simeq \prod_{1 \leqslant i \leqslant k} H_{i}$, where each $H_{i}$ acts weakly irreducibly on each $M_{p}^{i}$ and trivially on the $M_{p}^{j}$ for $j \neq i$;

(ii) $\mathcal{M}$ is isometric to the Riemannian product $\prod_{0 \leqslant i \leqslant k} \mathcal{M}_{i}$, where each $\mathcal{M}_{i}$ is the maximal integral leaf through $p$ of the parallel distribution $M^{i}$ generated by $M_{p}^{i}$; $\mathcal{M}_{0}$ is flat. 
If $(\mathcal{M}, g)$ is not supposed to be geodesically complete and simply connected, the same result holds, for the full holonomy group $H$ as well as for the restricted group $H^{0}$, except that the isometry of point (ii) is only local.

A consequence of this theorem in our situation is the following.

Proposition 5.3. Let $(\mathcal{M}, g)$ be a Riemannian or pseudo-Riemannian manifold and $p \in \mathcal{M}$. We suppose that the maximal trivial subspace $M_{p}^{0}$ of $H$ in $T_{p} \mathcal{M}$ is nondegenerate, and denote by $(\mathcal{M}, g) \simeq \prod_{0 \leqslant i \leqslant k}\left(\mathcal{M}_{i}, g_{i}\right)$ Wu's decomposition of $\mathcal{M}$.

Suppose that $(\mathcal{M}, g)$ admits a parallel and non-degenerate symplectic form $\omega$. Then $\omega_{i}$, the restriction of $\omega$ to $T \mathcal{M}_{i}$, is non-degenerate and

$$
(\mathcal{M}, g, \omega) \simeq \prod_{i}\left(\mathcal{M}_{i}, g_{i}, \omega_{i}\right)
$$

Proof. We use here the notation introduced in Wu's Theorem above. It is sufficient to show that the $M_{p}^{i}$ are pairwise $\omega$-orthogonal: the statement follows by parallel transport. Let us denote by $\Omega$ the element of $\mathfrak{s o}$ (ric) such that $\omega=g(\cdot, \Omega \cdot)$. By definition,

$$
M_{p}^{0}=\left\{x \in T_{p} \mathcal{M} ; H . x=\{x\}\right\} .
$$

So, with $x \in M_{p}^{0}$,

$$
\begin{aligned}
H \cdot \Omega(x) & =\Omega(H \cdot x) \quad(\Omega \text { is parallel, hence commutes with the action of } H) \\
& =\Omega(\{x\})=\{\Omega(x)\},
\end{aligned}
$$

therefore $\Omega(x) \in M_{p}^{0}$, hence $\Omega\left(M_{p}^{0}\right) \subset M_{p}^{0}$, so, as $\Omega$ is non-degenerate, $\Omega\left(M_{p}^{0}\right)=M_{p}^{0}$.

By point (i) of Wu's Theorem, for $i \geqslant 1$,

$$
M_{p}^{i}=\left(M_{p}^{0}\right)^{\perp} \cap\left\{x \in T_{p} \mathcal{M} ; \forall j \neq i, H_{j} . x=\{x\}\right\} .
$$

So, similarly, for each $i \geqslant 1, \Omega\left(M_{p}^{i}\right) \subset M_{p}^{0} \oplus M_{p}^{i}$. Now $\Omega \in \mathfrak{s o}$ (ric) so

$$
g\left(\Omega\left(M_{p}^{i}\right), M_{p}^{0}\right)=-g\left(M_{p}^{i}, \Omega\left(M_{p}^{0}\right)\right)=-g\left(M_{p}^{i}, M_{p}^{0}\right)=\{0\},
$$

and so $\Omega\left(M_{p}^{i}\right) \subset M_{p}^{i}$ (with equality). By definition of $\Omega$, the desired result follows.

So, Wu's holonomy decomposition provides a refinement of the Ricci decomposition given by the Main Theorem, at least a refinement of the decomposition of the factor on which ric is non-degenerate. Indeed, in this factor, ric, on the one hand, is parallel and non-degenerate, so is a (pseudo-)Riemannian metric, and, on the other hand, the trivial subspace of the action of the holonomy group is $\{0\}$, which is non-degenerate.

Corollary 5.4. Let $(\mathcal{M}, \omega, D)$ be a symplectic manifold with a symplectic connection $D$ the Ricci curvature of which is parallel and non-degenerate. Then $(\mathcal{M}, \omega, D)$ admits a unique decomposition into a Riemannian product (with respect to ric, considered as a metric), such that each factor is weakly irreducible. Moreover, this decomposition holds also for $\omega$ :

$$
(\mathcal{M}, g, \omega) \simeq \prod_{i}\left(\mathcal{M}_{i}, g_{i}, \omega_{i}\right)
$$

with $g$ standing here for ric, considered as the metric. 
Being unique and maximal, this decomposition is necessarily a refinement of that of the Main Theorem. Naturally, point (i) of the Main Theorem still applies and Ric is semi-simple on each factor (in fact, its minimal polynomial is one of the $P_{\lambda}$ ).

\subsection{A more precise description of the weakly irreducible factors}

Using the Main Theorem, we can now give a more precise description of the weakly irreducible subfactors given by Corollary 5.4. By the remark below, these factors are (pseudo-)Riemannian manifolds. We also introduce some vocabulary: paracomplex structures and related notions. Their names are chosen by analogy with the corresponding complex structures; other terminology is also used ('polarization' for a paracomplex structure, for example).

Important remark. On these subfactors, as ric is parallel and non-degenerate, ric is a (pseudo-)Riemannian metric and $D$ is its Levi-Civita connection. Moreover, such a manifold is obviously Einstein in that point of view, with Einstein constant 1. So, in the following, symplectic manifolds with a symplectic connection such that ric is parallel and non-degenerate will be viewed as Einstein non-Ricci flat manifolds admitting a parallel symplectic form.

Definition 5.5. A paracomplex structure on a manifold $\mathcal{M}$ of dimension $2 n$ is an endomorphism field $L$ on $\mathcal{M}$, integrable, satisfying $L^{2}=\mathrm{Id}$ with $\operatorname{dim} \operatorname{ker}(L-\mathrm{Id})=$ $\operatorname{dim} \operatorname{ker}(L+\mathrm{Id})$.

If $(\mathcal{M}, g)$ is pseudo-Riemannian, a paracomplex structure on $\mathcal{M}$ satisfying $g(L x, y)=$ $-g(x, L y)$ is said to be parahermitian. If, moreover, $D L=0$, it is said to be parakähler.

Remarks. A paracomplex structure therefore gives two complementary distributions of dimension $n: \operatorname{ker}(L \pm \mathrm{Id})$. Like $L$, these distributions are integrable. Equivalently, a paracomplex structure is given, up to sign, by the data of two such integrable distributions $E$ and $E^{\prime}: L= \pm\left(\operatorname{Id}_{E} \oplus-\operatorname{Id}_{E^{\prime}}\right)$.

For a parahermitian structure $L, \operatorname{ker}(L-\mathrm{Id})$ and $\operatorname{ker}(L+\mathrm{Id})$ are necessarily totally isotropic, so the signature of the metric is necessarily $(n, n)$.

Vocabulary. Let us also recall that a pseudo-Kähler manifold is a pseudo-Riemannian manifold $(\mathcal{M}, g)$ admitting a $g$-orthogonal parallel complex structure $J$ (in other words, a Kähler manifold with indefinite metric).

Now, a Riemannian or pseudo-Riemannian manifold admitting a parallel symplectic form is (pseudo-)Kähler or parakähler. The following proposition, using the Main Theorem $(\S 3)$, describes more precisely the situation when the manifold is Einstein non-Ricciflat. The matrices of the different involved objects are also given, to make the situation clearer for the reader.

Notation. For each integer $k, J_{k}$ will here denote the matrix

$$
\left(\begin{array}{cc}
0 & I_{k} \\
-I_{k} & 0
\end{array}\right)
$$


Proposition 5.6. Let $(\mathcal{M}, g)$ be a weakly irreducible Einstein non-Ricci-flat Riemannian or pseudo-Riemannian manifold and let $p \in \mathcal{M}$. We suppose $(\mathcal{M}, g)$ admits a parallel symplectic form $\omega$. Then, denoting $\operatorname{dim} \mathcal{M}$ by $2 n, \mathcal{M}$ is in one of the three following situations.

(i) $(\mathcal{M}, g)$ has a parakähler structure $L$ such that $\omega=\lambda g(\cdot, L \cdot)$ with some $\lambda$ in $\mathbb{R}^{*}$. In that case, $g$ is of signature $(n, n)$ and there is a basis of $T_{p} \mathcal{M}$ in which

$$
\operatorname{Mat}(g)=\left(\begin{array}{cc}
0 & I_{n} \\
I_{n} & 0
\end{array}\right), \quad \operatorname{Mat}(L)=\left(\begin{array}{cc}
-I_{n} & 0 \\
0 & I_{n}
\end{array}\right), \quad \operatorname{Mat}(\omega)=\lambda\left(\begin{array}{cc}
0 & I_{n} \\
-I_{n} & 0
\end{array}\right) .
$$

(ii) $(\mathcal{M}, g)$ has a (pseudo-)Kähler structure $J$ such that $\omega=\lambda g(\cdot, J \cdot)$ with some $\lambda$ in $\mathbb{R}^{*}$. In that case, $g$ is of signature $(2 p, 2 q)$ with $p+q=n$ and there is a basis of $T_{p} \mathcal{M}$ in which

$$
\operatorname{Mat}(g)=\left(\begin{array}{cc}
I_{2 p} & 0 \\
0 & -I_{2 q}
\end{array}\right), \quad \operatorname{Mat}(J)=\left(\begin{array}{cc}
J_{p} & 0 \\
0 & J_{q}
\end{array}\right), \quad \operatorname{Mat}(\omega)=\lambda\left(\begin{array}{cc}
J_{p} & 0 \\
0 & -J_{q}
\end{array}\right) .
$$

(iii) $(\mathcal{M}, g)$ has a pseudo-Kähler structure $J$ and a parakähler structure $L$ such that $J L=L J$ and such that $\omega=\alpha g(\cdot, L \cdot)+\beta g(\cdot, J \cdot)$ with $(\alpha, \beta) \in \mathbb{R}^{* 2}$. In that case, $n$ is even, $g$ is of signature $(n, n)$, and, setting $m=\frac{1}{2} n$, there is a basis of $T_{p} \mathcal{M}$ in which

$$
\begin{gathered}
\operatorname{Mat}(g)=\left(\begin{array}{cc}
0 & I_{2 m} \\
I_{2 m} & 0
\end{array}\right), \quad \operatorname{Mat}(L)=\left(\begin{array}{cc}
-I_{2 m} & 0 \\
0 & I_{2 m}
\end{array}\right), \quad \operatorname{Mat}(J)=\left(\begin{array}{cc}
J_{m} & 0 \\
0 & J_{m}
\end{array}\right), \\
\operatorname{Mat}(\omega)=\left(\begin{array}{cc}
0 & \alpha I_{2 m}+\beta J_{m} \\
-\alpha I_{2 m}+\beta J_{m} & 0
\end{array}\right) .
\end{gathered}
$$

Proof. After a possible rescaling, we may suppose that $g=$ ric. The decomposition (2.3), given in $\S 2(\mathrm{~d})$, of $T_{p} \mathcal{M}$ is stable under the action of $H$. So, by weak irreducibility of $\mathcal{M}$ and as ric is non-degenerate, the minimal polynomial of the endomorphism Ric is equal to a single factor $P_{\nu}^{\alpha_{\nu}}$ for some $\nu \in \mathbb{C}^{*}$ (with the definition given in (2.2), §2). By point (i) of the Main Theorem in $\S 3, \alpha_{\nu}=1$.

Let us discuss the situation for the different possible values of $\nu$.

(i) If $\nu$ is real. Let us set $L=(1 / \nu)$ Ric; $L$ is a parallel endomorphism of $\mathfrak{s o}$ (ric) with minimal polynomial $(X-1)(X+1)$, as $\alpha_{\nu}=1$. If $x, y \in \operatorname{ker}(L-\varepsilon$ Id $)$ with $\varepsilon= \pm 1$, $\operatorname{ric}(x, y)=\varepsilon \operatorname{ric}(x, L y)=-\varepsilon \operatorname{ric}(L x, y)=-\operatorname{ric}(x, y)$ so $\operatorname{ker}(L-\mathrm{Id})$ and $\operatorname{ker}(L+\mathrm{Id})$ are both ric-totally isotropic (that remark is also contained in Lemma 2.4, $\S 2(\mathrm{~d})$ ). As $T_{p} \mathcal{M}=\operatorname{ker}(L-\mathrm{Id}) \oplus \operatorname{ker}(L+\mathrm{Id})$ and ric is non-degenerate, these two spaces are of dimension $n$ and ric is of signature $(n, n)$; so $L$ is a parakähler structure. Finally, there is a basis of $T_{p} \mathcal{M}$ as announced in the proposition, with $\lambda=(1 / \nu)$, and $\omega=$ $\operatorname{ric}\left(\cdot, \operatorname{Ric}^{-1} \cdot\right)=\lambda \operatorname{ric}\left(\cdot, L^{-1} \cdot\right)=\lambda \operatorname{ric}(\cdot, L \cdot)$ as $L=L^{-1}$. 
(ii) If $\nu$ is purely imaginary. Let us set $J=-(1 /|\nu|)$ Ric; $J$ is a parallel endomorphism of $\mathfrak{s o}$ (ric) with minimal polynomial $(X-\mathrm{i})(X+\mathrm{i})=X^{2}+1$, as $\alpha_{\nu}=1$, so $J^{2}=-\mathrm{Id}$ and $J$ is a Kähler or pseudo-Kähler structure (whether ric is definite or not). By the same computation as above or by Lemma 2.4, and extending ric to a bilinear complex form on $T_{p} \mathcal{M} \otimes \mathbb{C}, \operatorname{ker}(J-\mathrm{i} I d)$ and $\operatorname{ker}(J+\mathrm{i} I d)$ are both ric-totally isotropic; let $n$ be their dimension $(\mathcal{M}$ is then of dimension $2 n)$. The complex conjugation $e \mapsto \bar{e}$ being a linear isomorphism of $\operatorname{ker}(J-\mathrm{i}$ Id $)$ to $\operatorname{ker}(J+\mathrm{i}$ Id $)$ and ric being non-degenerate, the sesquilinear form $h:\left(e, e^{\prime}\right) \mapsto \operatorname{ric}\left(e, \bar{e}^{\prime}\right)$ is non-degenerate on $\operatorname{ker}(J-\mathrm{i} I d)$ and on $\operatorname{ker}(J+\mathrm{i} I d)$. Its signature on each of these spaces is the same, let us denote it by $(p, q)$. So if $\left(e_{i}\right)_{i=1}^{n}$ is an $h$-(pseudo-)orthonormal basis of $\operatorname{ker}(J-\mathrm{i} \mathrm{Id})$, and setting $\beta=\left(\left(e_{i}\right)_{i=1}^{n}\left(\overline{e_{i}}\right)_{i=1}^{n}\right)$,

$$
\operatorname{Mat}_{\beta}(\text { ric })=\left(\begin{array}{cc}
0 & I_{p, q} \\
I_{p, q} & 0
\end{array}\right) \quad \text { and } \quad \operatorname{Mat}_{\beta}(J)=\left(\begin{array}{cc}
\mathrm{i} I & 0 \\
0 & -\mathrm{i} I
\end{array}\right) .
$$

Now in the real basis $\left(f_{i}, f_{i}^{\prime}\right)_{i=1}^{n}$ of $T_{p} \mathcal{M}$ defined by

$$
f_{i}=\frac{1}{\sqrt{2}}\left(e_{i}+\bar{e}_{i}\right) \quad \text { and } \quad f_{i}^{\prime}=\frac{1}{\mathrm{i} \sqrt{2}}\left(e_{i}-\bar{e}_{i}\right)
$$

the matrices of ric, $J$ and $\omega$ have the announced form, with $\lambda=(1 /|\nu|)$. Besides, $\omega=\operatorname{ric}\left(\cdot, \operatorname{Ric}^{-1} \cdot\right)=\lambda \operatorname{ric}(\cdot, J \cdot)$ as $J=-J^{-1}$.

(iii) Otherwise. Let us set

$$
L=\frac{1}{2 \operatorname{Re} \nu}\left(\operatorname{Ric}+|\nu|^{2} \operatorname{Ric}^{-1}\right) \quad \text { and } \quad J=\frac{1}{2 \operatorname{Im} \nu}\left(\operatorname{Ric}-|\nu|^{2} \operatorname{Ric}^{-1}\right) .
$$

As $\alpha_{\nu}=1$, a short computation gives

$$
L^{2}-\mathrm{Id}=J^{2}+\mathrm{Id}=P_{\nu}(\mathrm{Ric})=0 .
$$

As in the previous case, using the non-degenerate hermitian form $h: e \mapsto \operatorname{ric}(e, \bar{e})$ of $T_{p} \mathcal{M} \otimes \mathbb{C}$ and the fact that $J$ and $L$ commute, and denoting by $n$ the dimension of $\operatorname{ker}(L-\mathrm{Id}) \cap \operatorname{ker}(J-\mathrm{i} \mathrm{Id})$, we obtain a basis $\left(e_{i}\right)_{i=1}^{2 m}$ of $\operatorname{ker}(J-\mathrm{i} \mathrm{Id})$ such that, setting $\beta=\left(\left(e_{i}\right)_{i=1}^{m},\left(\overline{e_{i}}\right)_{i=1}^{m},\left(\overline{e_{i}}\right)_{i=m+1}^{2 m},\left(e_{i}\right)_{i=m+1}^{2 m}\right)$,

$$
\begin{gathered}
\operatorname{Mat}_{\beta}(\text { ric })=\left(\begin{array}{cc}
0 & I_{2 m} \\
I_{2 m} & 0
\end{array}\right), \quad \operatorname{Mat}_{\beta}(L)=\left(\begin{array}{ccc}
-I_{2 m} & 0 \\
0 & I_{2 m}
\end{array}\right), \\
\operatorname{Mat}_{\beta}(J)=\left(\begin{array}{cccc}
\mathrm{i} I_{m} & 0 & 0 & 0 \\
0 & -\mathrm{i} I_{m} & 0 & 0 \\
0 & 0 & -\mathrm{i} I_{m} & 0 \\
0 & 0 & 0 & \mathrm{i} I_{m}
\end{array}\right) .
\end{gathered}
$$

$\mathcal{M}$ is of dimension $4 m$ and ric of signature $(2 m, 2 m)$. As $L$ and $J$ are, moreover, in $\mathfrak{s o}(\text { ric })^{\mathfrak{h}}$, they are then, respectively, a parakähler and a pseudo-Kähler structure on $(\mathcal{M}$, ric $)$. Note also that they commute. 
Now in the real basis $\left(\left(f_{i}\right)_{i=1}^{n},\left(f_{i}^{\prime}\right)_{i=1}^{n},\left(f_{i}\right)_{i=n+1}^{2 n},\left(f_{i}^{\prime}\right)_{i=n+1}^{2 n}\right)$ of $T_{p} \mathcal{M}$ defined by

$$
f_{i}=\frac{1}{\sqrt{2}}\left(e_{i}+\overline{e_{i}}\right) \quad \text { and } \quad f_{i}^{\prime}=\frac{1}{\mathrm{i} \sqrt{2}}\left(e_{i}-\overline{e_{i}}\right),
$$

the matrices of ric, $L, J$ and $\omega$ have the announced form, with $\alpha+\mathrm{i} \beta=(1 / \nu)$. Besides, $\omega=\alpha \operatorname{ric}(\cdot, L \cdot)+\beta \operatorname{ric}(\cdot, J \cdot)$.

\section{Some remarks and examples}

\subsection{An example with $\operatorname{Ric}^{2}=0$ and Ric $\neq 0$}

The Main Theorem requires that ric is non-degenerate to ensure that Ric has no nilpotent part. This assumption is necessary; it can be seen in a very simple example borrowed from $\left[\mathbf{7}\right.$, p. 40]. Take $(\mathcal{M}, \omega)=\left(\mathbb{R}^{2}, \mathrm{~d} x \wedge \mathrm{d} y\right)$ and, denoting the coordinate vectors by $X$ and $Y$, the connection defined by

$$
D_{X} X=D_{Y} X=D_{X} Y=0, \quad D_{Y} Y=x X .
$$

In particular, $X$ is stable by holonomy. By definition, $D$ is torsion free and we check that

$$
\begin{aligned}
\left(D_{a X+b Y} \omega\right)(X, Y) & =a\left(D_{X} \omega\right)(X, Y)+b\left(D_{Y} \omega\right)(X, Y) \\
& =a\left[L_{X}(\omega(X, Y))-\omega\left(X, D_{X} Y\right)\right]+b\left[L_{Y}(\omega(X, Y))-\omega\left(X, D_{Y} Y\right)\right] \\
& =0
\end{aligned}
$$

so $D$ is symplectic. Now $R(X, Y) X=0$ and $R(X, Y) Y=-X$, so $\operatorname{ric}(Y, Y)=-1$, ker ric $=\operatorname{span}(X)$ and $D$ ric $=0$. Actually, $D R=0$, i.e. $(\mathcal{M}, \omega, D)$ is even symmetric. Now, $\operatorname{Ric}(X)=0$ and $\operatorname{Ric}(Y)=X$ so $\operatorname{Ric} \neq 0$ and $\operatorname{Ric}^{2}=0$.

Remark. Examples where the minimal polynomial $P_{\lambda}$ of Ric corresponds to a $\lambda$ in $\mathbb{R}^{*}, i \mathbb{R}^{*}$ or $\mathbb{C} \backslash\left(\mathbb{R}^{*} \cup \mathrm{i} \mathbb{R}^{*}\right)$ are numerous. They are the parakähler and (pseudo-)kähler manifolds (see Proposition 5.6 in §5.2). The next subsection gives symmetric examples of the three types.

\subsection{The low-dimensional cases}

Let us recall the following fact.

Proposition 6.1. Let $(\mathcal{M}, g)$ be

- either a Riemannian or pseudo-Riemannian manifold of dimension three or less, or

- a 'complex Riemannian' manifold (i.e. a complex manifold with a complex bilinear — warning: not sesquilinear — symmetric form $g$ ) of complex dimension three or less,

then the (real) curvature tensor $R$ of $(\mathcal{M}, g)$ is a linear function of ric. 
Table 1.

\begin{tabular}{cccc}
\hline space & dimension & $\mathfrak{s o}(g)^{\mathfrak{h}}$ & $\operatorname{sgn}($ ric $)$ \\
\hline$S L(2, \mathbb{R}) / \mathbb{R}^{*}$ & 2 & $\mathbb{R} L$ & $(1,1)$ \\
$S U(2) / S O(2)$ & 2 & $\mathbb{R} J$ & $(2,0)$ \\
$S L(2, \mathbb{R}) / S O(2)$ & 2 & $\mathbb{R} J$ & $(0,2)$ \\
$S L(2, \mathbb{C}) / \mathbb{C}^{*}$ & 4 & $\mathbb{R} L+\mathbb{R} J$ & $(2,2)$ \\
\hline
\end{tabular}

Table 2 .

\begin{tabular}{cccc}
\hline space & dimension & $\mathfrak{s o}(g)^{\mathfrak{h}}$ & sgn(ric) \\
\hline$S L(3, \mathbb{R}) /\left(S L(2, \mathbb{R}) \times \mathbb{R}^{*}\right)$ & 4 & $\mathbb{R} L$ & $(2,2)$ \\
$S U(3) /(S U(2) \times S O(2))$ & 4 & $\mathbb{R} J$ & $(4,0)$ \\
$S U(1,2) /(S U(1,1) \times S O(2))$ & 4 & $\mathbb{R} J$ & $(2,2)$ \\
$S U(1,2) /(S U(2) \times S O(2))$ & 4 & $\mathbb{R} J$ & $(0,4)$ \\
\hline
\end{tabular}

A proof can be found in [1, pp. 47-49]. Consequently, a Ricci-parallel manifold of low enough dimension, as required in the above proposition, is locally symmetric. So in the Main Theorem, the weakly indecomposable subfactors of the factor on which ric is non-degenerate are (locally) symmetric as soon as

(i) they are of dimension two, or

(ii) they are of dimension four and admit a pseudo-Kähler structure $J$ and a parakähler structure $L$; in this case, indeed, $L J=J L$ defines on $\mathcal{M}$ a complex structure with respect to which the complex form $h(\cdot, \cdot)=g(\cdot, \cdot)-\mathrm{i} g(\cdot, L J \cdot)$ is $\mathbb{C}$-bilinear, symmetric (besides, after Proposition 5.6 of $\S 5.2$, manifolds of this type are of (real) dimension multiple of four, so dimension six is here not relevant).

Then Berger's list - you can find its restriction to the symplectic case, with which we deal here, in [3, pp. 267, 268] — provides the list of the relevant simply connected symmetric spaces. We give each time the structure of the algebra $\mathfrak{s o}(g)^{\mathfrak{h}}$ of the parallel endomorphisms, with the following convention: $L$ denotes a parakähler structure $\left(L^{2}=\mathrm{Id}\right), J$ a (pseudo-)Kähler structure $\left(J^{2}=-\mathrm{Id}\right)$. The spaces are denoted in Table 1 (see [2, p. 315]).

Remark. To obtain the full list of the simply connected, simple, symplectic symmetric spaces of dimension four or less, one has to add the ones of dimension four and with $\mathfrak{s o}(g)^{\mathfrak{h}}=\mathbb{R} L$ or $\mathfrak{s o}(g)^{\mathfrak{h}}=\mathbb{R} J$ (see Table 2 ).

Acknowledgements. I thank M. Cahen for his quick answer to a technical question and the referee for his careful reading of the manuscript, which has helped me to make it clearer. 


\section{References}

1. A. L. Besse, Einstein manifolds (Springer, 1987).

2. P. Bieliavsky, Four-dimensional simply connected symplectic symmetric spaces, Geom. Dedicata 69 (1998), 291-316.

3. P. Bieliavsky, Semi-simple symplectic symmetric spaces, Geom. Dedicata 73 (1998), 245-273.

4. P. Bieliavsky, M. Cahen and S. Gutt, A class of homogeneous symplectic manifolds, in Geometry and nature (ed. H. Nencka and J.-P. Bourguignon), Contemporary Mathematics, vol. 203, pp. 241-255 (American Mathematical Society, Providence, RI, 1997).

5. Ch. Boubel and L. Bérard Bergery, On pseudo-Riemannian manifolds whose Ricci tensor is parallel, Geom. Dedicata 86 (2001), 1-18.

6. F. Bourgeois And M. CAhen, A variational principle for symplectic connections, $J$. Geom. Phys. 30 (1999), 233-265.

7. M. Cahen, S. Gutt and J. Rawnsley, Symplectic connections with parallel Ricci tensor, in Poisson geometry, pp. 31-41 (Banach Center Publications, Warsaw, 2000).

8. S. Kobayashi and K. Nomizu, Foundations of differential geometry, vol. I (Interscience, 1969).

9. A. Lichnerowicz, Quantum mechanics and deformations of geometrical dynamics, in Quantum theory, groups, fields and particles, pp. 3-82 (Reidel, Dordrecht, 1983).

10. O. Loos, Symmetric spaces (Benjamin, New York, 1969).

11. J. Milnor, Morse theory (Princeton University Press, Princeton, NJ, 1963).

12. H. Wu, Holonomy groups of indefinite metrics, Pac. J. Math. 20 (1967), 351-392. 